

\title{
An axiomatic characterization of different majority concepts.
}

\author{
Thierry Marchant \\ Thierry.Marchant@UGent . be
}

Ghent, December 2005

\begin{abstract}
A lot of decision support systems use some kind of aggregation procedure based on the concept of majority, but not always the same one; it can be simple majority, weak majority or one of the many other kinds of majority. This paper attempts to present the main variants of majority and to characterize them in a uniform way. Consequently, it is now easier to compare different kinds of majority and to understand the dissimilarities (or similarities) between them. This should help decision analysts willing to use a majority procedure to choose the right one for their problem and context.
\end{abstract}

Keywords: Multi-Criteria Decision Aiding, Group Decision Support Systems, ElECTRE I, TACTIC, weak majority, simple majority

\section{Introduction}

Many aggregation procedures used in multicriteria decision aiding or group decision support are based on some majority concept: for instance, the various EleCtre methods [16], Melchior [10], PROCFTn [2], TACTIC [19], Vikor [14] and VIP-G [5]. See also, among others, $[4,7,9,11,13]$ for some recent but unnamed methods. The majority concepts used in these papers are not always the same: some papers use simple majority, others use absolute majority, weak majority or other variants of majority. Of course, these various majority concepts are not equivalent and can sometimes yield very different results. This prompts some important questions. Do we have good reasons to use different kinds of majority in ElECTRE, TACTIC and VIP-G? Wouldn't Melchior or ViKor work better with another kind of majority?

Understanding how these majority-based procedures work and what makes each one different from the others is therefore crucial if we want to use a procedure that is adequate for the problem we want to handle. That is why we present in this paper various characterizations of some major majority concepts.

*I wish to thank Denis Bouyssou for his comments on a previous version of this paper. 
Some of the majority concepts described in this paper have been studied earlier $[1,3,8,12,17$, among others] in the framework of Social Choice Theory, but most of the time in the context of pairwise elections where a proposition is opposed to the status quo. In this context, the proposition passes if it has a majority (of some type); but if the proposition does not have a majority, then the status quo wins, even if the status quo is not supported by a majority. Consequently, there is always a winner and, sometimes, two (when the proposition and the status quo are supported by a majority).

In the context of multicriteria decision aiding or group decision support, very often, there is no status quo: all alternatives play the same role. So, if none of two alternatives has a majority, they can be declared incomparable. Hence, the majority procedures that we will study in this paper will often be different from those previously studied in Social Choice Theory, even if they have some features in common.

Another difference between the analysis of aggregation procedures in the context of multicriteria decision aiding and Social Choice Theory is that, in the latter, abstention is sometimes taken into account [6] while, in multicriteria decision aiding, abstention is hardly relevant. Indeed, a voter can choose not to vote; in that case, we have no preference relation for that voter. But, in multicriteria decision aiding, for each criterion, we always have a preference relation, even if it is not complete.

In Section 2, we formally define the various majority concepts that are characterized in Section 3 (where the size of the set of criteria is fixed) and 4 (where the size of the set of criteria is allowed to vary). Section 5 concludes.

\section{Notation and definitions}

The set of potential criteria is denoted by $\mathbb{N}=\{1,2, \ldots\}$ and the set of alternatives, finite, by $X=\{x, y, \ldots\}$. The set of criteria that is actually used is denoted by $N=\{1,2, \ldots, n\}$. For each criterion $i$, we suppose that we have a complete preference relation on $X$, denoted by $\succsim_{i}$. This preference relation is not supposed to have any particular property but completeness; for instance, it is not necessarily transitive. The asymmetric and symmetric parts of $\succsim_{i}$ are denoted by $\succ_{i}$ and $\sim_{i}$. A profile of size $n$ is defined as a $n$-tuple $p=\left(\succsim_{1}, \succsim_{2}, \ldots, \succsim_{n}\right)$. It can be used to represent the preferences of a decisionmaker according to $n$ criteria (or the preferences of $n$ decision-makers). The set of all complete binary relations on $X$ is denoted by $\mathcal{C}$. The set of all possible profiles of size $n$ is therefore the $n$-fold cartesian product $\mathcal{C}^{n}$. The set of all binary relations on $X$ is denoted by $\mathcal{R}$. We define a $n$-aggregation procedure (or just $n$-procedure) as a mapping $\succsim^{n}: \mathcal{C}^{n} \rightarrow \mathcal{R}: p \rightarrow \succsim^{n}(p)$. An aggregation procedure (or just procedure) is an infinite collection of $n$-aggregation procedures, for $n \in \mathbb{N}$. It is denoted by $\succsim=\left\{\succsim^{1}, \succsim^{2}, \ldots\right\}$. The asymmetric and symmetric parts of $\succsim^{n}(p)$ are denoted by $\stackrel{n}{\succ}^{\prime}(p)$ and $\stackrel{n}{\sim}(p)$. We now present some of the most important variants of majority. Because there are no standard names for aggregation procedures, I use my own terminology but I nevertheless try to follow 
some tradition.

\section{Weak majority}

$$
x \succsim^{n}(p) y \Leftrightarrow \#\left\{i \in N: x \succsim_{i} y\right\} \geq\lceil n / 2\rceil,
$$

with $\lceil t\rceil$ the smallest integer that is not smaller than $t$ (upwards rounding). This $n$-procedure can be presented in a different but perfectly equivalent way [8]:

$$
x \succ^{n}(p) y \Leftrightarrow \#\left\{i \in N: x \succ_{i} y\right\}>n / 2,
$$

with $\succsim^{n}(p)$ complete for all $p$. It is sometimes called absolute majority.

\section{Qualified weak majority}

$$
x \succsim^{n}(p) y \Leftrightarrow \#\left\{i \in N: x \succsim_{i} y\right\} \geq \delta_{n},
$$

with $\delta_{n}$ integer and $0<\delta_{n} \leq\lceil n / 2\rceil$. An equivalent definition is:

$$
x \iota^{n}(p) y \Leftrightarrow \#\left\{i \in N: x \succ_{i} y\right\}>\delta_{n},
$$

with $\succsim^{n}(p)$ complete for all $p, \delta_{n}$ integer, $\lfloor n / 2\rfloor \leq \delta_{n}<n$ and $\lfloor t\rfloor$ the largest integer that is not larger than $t$ (downwards rounding). This is closely related to what [8] calls absolute special majority (in the context of pairwise elections with a status quo). It is also sometimes called qualified majority.

\section{Electre I majority}

$$
x \succsim^{n}(p) y \Leftrightarrow \#\left\{i \in N: x \succsim_{i} y\right\} \geq \delta_{n},
$$

with $\delta_{n}$ integer and $\lceil n / 2\rceil \leq \delta_{n} \leq n$. Note that with this $n$-procedure, there can be two alternatives $x$ and $y$ such that NOT $x \succsim^{n}(p) y$ and NOT $y \succsim^{n}(p) x$. We then say that $x$ and $y$ are incomparable in the profile $p$. We call this $n$-procedure 'ELECTRE I majority' because, if we omit the weights and the vetoes, it is the one used in Electre I [15].

\section{Generalized qualified weak majority}

$$
x \succsim^{n}(p) y \Leftrightarrow \#\left\{i \in N: x \succsim_{i} y\right\} \geq \delta_{n},
$$

with $\delta_{n}$ integer and $0<\delta_{n} \leq n$. Note that ELECTRE I, weak majority and qualified weak majority are special cases of generalized qualified weak majority.

\section{Simple majority}

$$
x \succsim^{n}(p) y \Leftrightarrow \#\left\{i \in N: x \succsim_{i} y\right\} \geq \#\left\{i \in N: y \succsim_{i} x\right\} .
$$

This $n$-procedure is sometimes presented in a different but perfectly equivalent way $[8]$ :

$$
x \succsim^{n}(p) y \Leftrightarrow \#\left\{i \in N: x \succ_{i} y\right\} \geq \#\left\{i \in N: y \succ_{i} x\right\} .
$$




\section{a-Qualified simple majority}

$$
x \succsim^{n}(p) y \Leftrightarrow \#\left\{i \in N: x \succsim_{i} y\right\} \geq \#\left\{i \in N: y \succsim_{i} x\right\}+\delta_{n},
$$

with $\delta_{n}$ integer and $-n<\delta_{n} \leq n$. We call this procedure a-qualified simple majority to make a distinction with another generalization of simple majority that we present in the next paragraph. The "a" in "a-qualified simple majority" stands for additive, because the threshold $\delta_{n}$ is added.

\section{m-Qualified simple majority}

$$
x \succsim^{n}(p) y \Leftrightarrow \#\left\{i \in N: x \succsim_{i} y\right\} \geq \delta_{n} \#\left\{i \in N: y \succsim_{i} x\right\},
$$

with $0<\delta_{n} \leq n$ and $k \delta_{n}$ in $N$ for some $k$ in $N$. The "m" in "m-qualified simple majority" stands for multiplicative, because the threshold $\delta_{n}$ is multiplied.

\section{Tactic majority}

$$
x \iota^{n}(p) y \Leftrightarrow \#\left\{i \in N: x \succ_{i} y\right\}>\delta_{n} \#\left\{i \in N: y \succ_{i} x\right\},
$$

with $\succsim^{n}(p)$ asymmetric for all $p, 1 \leq \delta_{n}<n$ and $k \delta_{n}$ in $N$ for some $k$ in $N$. We call this $n$-procedure 'TACTIC majority' because, if we omit the weights and the vetoes, it is the one used in TACTIC [19]. Remark that we cannot generalize this $n$-procedure by considering thresholds $\delta$ smaller than 1 . It would lead to the impossible conclusion that $x \succ^{n}(p) x$, for any $x$ and any profile $p$. We therefore introduce a slightly modified version of TACTIC majority in the next paragraph.

\section{r-Tactic majority}

$$
x \succsim^{n}(p) y \Leftrightarrow \#\left\{i \in N: x \succ_{i} y\right\} \geq \delta_{n} \#\left\{i \in N: y \succ_{i} x\right\},
$$

with $1 \leq \delta_{n} \leq n$ and $k \delta_{n}$ in $N$ for some $k$ in $N$. With this $n$-procedure, we always have $x \stackrel{n}{\sim}(p) y$ when $x \sim_{i} y$ for all criteria. In particular, $x \sim^{n}(p) x$ (reflexivity). It is the only difference with TACTIC. For any $x \neq y$ and $\delta_{n}>1$, both $n$-procedures always yield the same result.

\section{Generalized r-Tactic majority}

$$
x \succsim^{n}(p) y \Leftrightarrow \#\left\{i \in N: x \succ_{i} y\right\} \geq \delta_{n} \#\left\{i \in N: y \succ_{i} x\right\},
$$

with $0<\delta_{n} \leq n$ and $k \delta_{n}$ in $N$ for some $k$ in $N$. This is closely related to what [8] calls relative special majority (in the context of pairwise elections with a status quo).

The reason why we impose that $\delta_{n}$ be integer or that $k \delta_{n}$ be in $N$ for some $k \in N$ is that, if we do not, then several values of $\delta_{n}$ can correspond to the same $n$-procedure. This would complicate the proofs. For example, the qualified weak 
majority for $n=10$ and a threshold equal to 3.2 is equivalent to the qualified weak majority for $n=10$ and a threshold equal to 4 .

We now present two conditions that are clearly satisfied by all the above defined $n$-procedures and that will allow us to use a simple graphical representation.

A 1 Independence of Irrelevant Alternatives. Suppose $p$ and $p^{\prime}$ are two profiles such that, for some alternatives $x$ and $y$ and for all criteria $i$ in $N$,

$$
x \succsim_{i} y \Leftrightarrow x \succsim_{i}^{\prime} y \text { and } y \succsim_{i} x \Leftrightarrow y \succsim_{i}^{\prime} x .
$$

Then

$$
x \succsim^{n}(p) y \Leftrightarrow x \succsim^{n}\left(p^{\prime}\right) y \quad \text { and } \quad y \succsim^{n}(p) x \Leftrightarrow y \succsim^{n}\left(p^{\prime}\right) x .
$$

A 2 Anonymity. Suppose $\sigma$ is a permutation of $N$. We denote by $\sigma(p)$ a profile identical to $p$ except that the order of the relations has been changed according to $\sigma$. Anonymity imposes that $\succsim^{n}(p)=\succsim^{n}(\sigma(p))$.

The first condition states that the global preference between two alternatives should depend only on the single-criteria preferences between these two alternatives. Other alternatives cannot play any role. The second condition imposes that all criteria play exactly the same role. So, what they represent or their name is irrelevant; whence anonymity. This condition is also sometimes called symmetry.

For all $n$-procedures satisfying Independence of Irrelevant Alternatives and Anonymity, the relation between two alternatives $x$ and $y$ in the global relation $\succsim^{n}(p)$ depends only on $\alpha$, the number of criteria $i$ such that $x \succ_{i} y, \beta$, the number of criteria such that $x \sim_{i} y$ and $\gamma$, the number of criteria such that $y \succ_{i} x$. So, any profile can be represented as a point with coordinates $(\alpha, \beta, \gamma)$ in a three-dimensional space. Remark that the sum of $\alpha, \beta$ and $\gamma$ is $n$ and, so, given $n$ the number of criteria, each profile is a point lying in the plane defined by $\alpha+\beta+\gamma=n$. Because $\alpha, \beta, \gamma \geq 0$, all possible profiles lie in a simplex, i.e. the equilateral triangle with vertices $(n, 0,0),(0, n, 0)$ and $(0,0, n)$. We can therefore represent the outcome between two alternatives in a simplex, in function of these three numbers (see Fig.1, left). An analogous representation has been used by $[17,20,18]$. Only the points with integer coordinates correspond to possible profiles; they form a kind of lattice. Fig. 2 is a graphical representation of the majority concepts presented above.

\section{Characterization of some $n$-aggregation pro- cedures}

In this section, I present some characterizations of $n$-aggregation procedures. Contrary to the next section, none of the axioms used in this section involve profiles of different size. Before turning to the characterizations of some $n$ procedures, I present some conditions that are satisfied by all $n$-procedures defined in Section 2. 

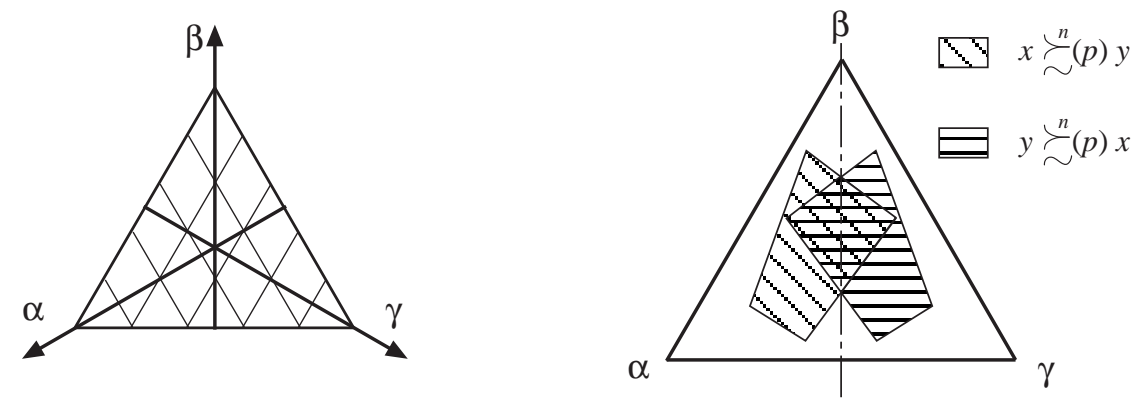

Figure 1: Left: the simplex for a 5-aggregation procedure. In this figure, the axes and the lattice of integer coordinates are represented but will be omitted in the rest of the paper. Right: geometrical representation of a neutral $n$-aggregation procedure. The profiles lying in the area with oblique hatching yield $x \succsim^{n}(p) y$. The profiles lying in the area with horizontal hatching yield $y \succsim^{n}(p) x$. The profiles located where both areas overlap yield $x \succsim^{n}(p) y$ and $y \succsim^{n}(p) x$, i.e. $x \sim^{n}(p) y$. The profiles lying in none of these areas yield ' $x$ and $y$ are incomparable'.

If $\pi$ is a permutation of $X$, we denote by $\pi(p)$ the profile that is obtained from $p$ by permuting all alternatives in each relation $\succsim_{i}$ according to $\pi$. Similarly, $\pi\left(\succsim^{n}(p)\right)$ denotes the relation obtained from $\succsim^{n}(p)$ by permuting all alternatives according to $\pi$.

A 3 Neutrality. Suppose $\pi$ is a permutation of $X$. Then, $\succsim^{n}(\pi(p))=\pi\left(\succsim^{n}(p)\right)$.

This excludes the existence of a status quo that would be treated in a different way as the other alternatives.

If we impose Independence of Irrelevant Alternatives and Anonymity, then Neutrality can easily be given a geometrical representation in the simplex. The area where $y \succsim^{n}(p) x$ is the reflection of the area where $x \succsim^{n}(p) y$ through a vertical mirror (see Fig.1, right). Furthermore, the shape of this area is independent of the pair $x, y$.

A 4 Weak Non-Negative Responsiveness 1. Suppose $p$ and $p^{\prime}$ are two identical profiles except that there is a criterion $i$ and two alternatives $x$ and $y$ such that $y \succ_{i} x$ and $x \succ_{i}^{\prime} y$. If $x \succsim^{n}(p) y$, then $x \succsim^{n}\left(p^{\prime}\right) y$.

This condition is very compelling for it imposes that turning a single-criterion preference $y \succ_{i} x$ into $x \succ_{i}^{\prime} y$ (thus improving the position of $x$ w.r.t. $y$ ) cannot lead to a deterioration of the position of $x$ w.r.t. $y$ in the global preference relation.

Under Independence of Irrelevant Alternatives and Anonymity, Weak NonNegative Responsiveness 1 implies that, if $x \succsim^{n}(p) y$ and if we move West on a horizontal line, then we still have $x \succsim^{n}\left(p^{\prime}\right) y$ (see Fig.3).

I now define a very simple and mild condition. 


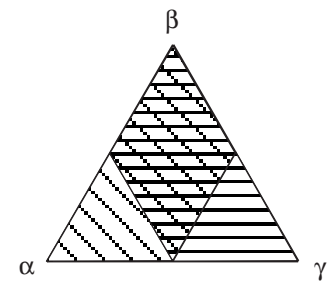

Weak majority, $\delta=n / 2$

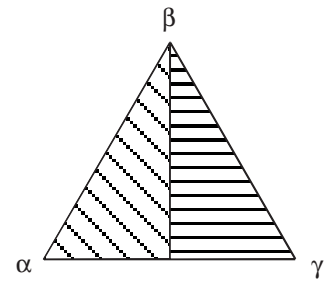

Simple majority, $\delta=0$

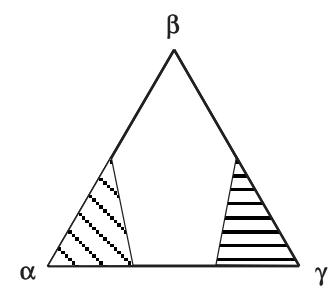

m-Qualified simple majority, $\delta>1$

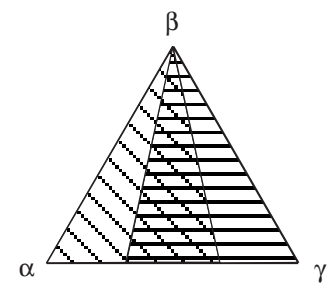

Generalized r-Tactic, $\delta<1$

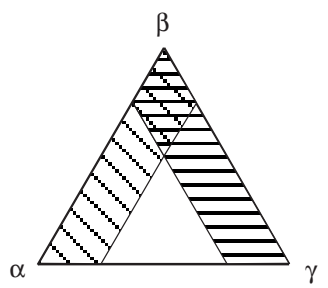

Electre, $\delta>n / 2$

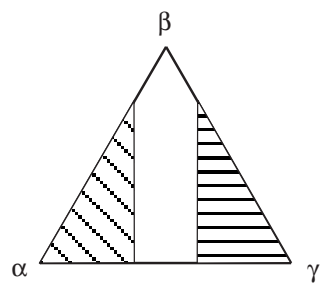

a-Qualified simple majority, $\delta>0$

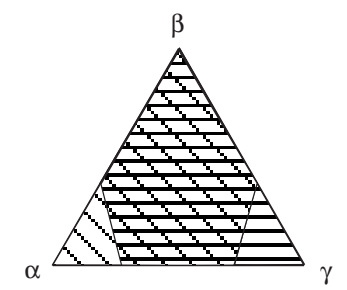

m-Qualified simple majority, $\delta<1$

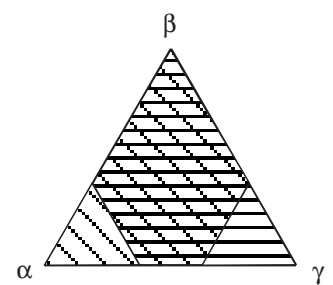

Qualified weak majority, $\delta<n / 2$

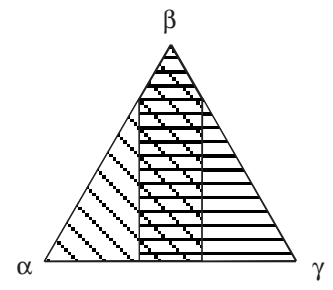

a-Qualified simple majority, $\delta<0$

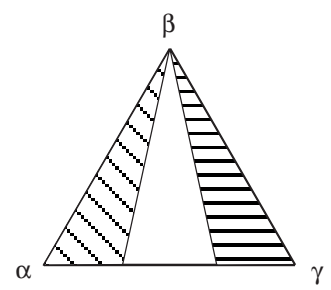

Tactic, $\delta>1$

Figure 2: Graphical representations of different majority concepts 

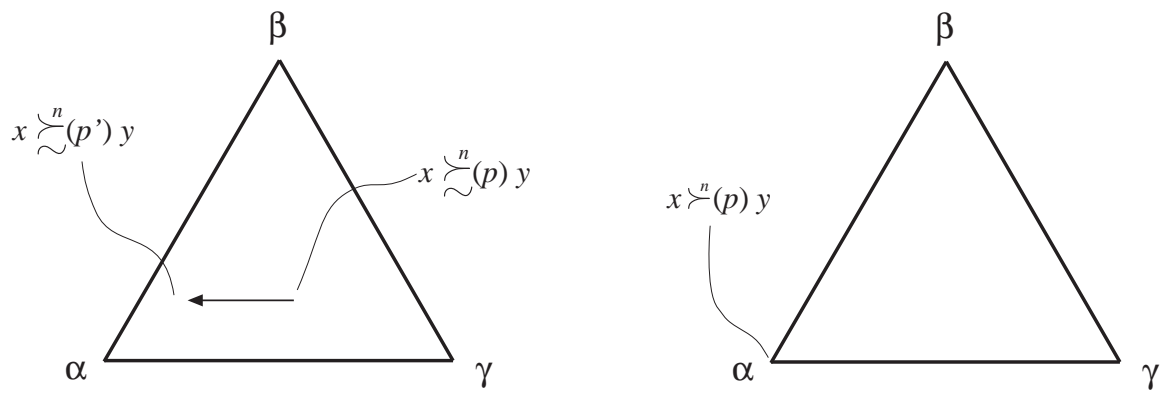

Figure 3: Geometrical representation of Weak Non-Negative Responsiveness 1 (left) and Unanimity (right)

A 5 Unanimity. If $x \succ_{i} y$, for all $i$ in $N$, then $x \succ^{n}(p) y$.

Under Independence of Irrelevant Alternatives and Anonymity, Unanimity implies that the West vertex of the simplex is a point where $x \succ^{n}(p) y$ (see Fig.3).

\subsection{The generalized qualified weak majority and some spe- cial cases}

A key property of all $n$-procedures in this family is

A 6 Limited Influence of Indifference. Suppose $p$ and $p^{\prime}$ are two identical profiles except that there is a criterion $i$ and two alternatives $x$ and $y$ such that $x \succ_{i} y$ and $x \sim_{i}^{\prime} y$. Then $x \succsim^{n}(p) y$ iff $x \succsim^{n}\left(p^{\prime}\right) y$.

The intuition for this condition is the following. Even if there is less support for the global strict preference (i.e. $\succ^{n}$ ) of $x$ over $y$ in $p^{\prime}$ than in $p$, we might consider that there is not less support for the global weak preference (i.e. $\succsim^{n}$ ) of $x$ over $y$ in $p^{\prime}$ than in $p$. It is then reasonable to impose that $x \succsim^{n}\left(p^{\prime}\right) y$ and this is precisely what Limited Influence of Indifference does.

Suppose now that $x \succ^{n}(p) y$. Then, because there is less support for the global strict preference of $x$ over $y$ in $p^{\prime}$ than in $p$, it might happen that $x \sim \sim^{n}\left(p^{\prime}\right) y$. This is not prevented by Limited Influence of Indifference. So, moving from $x \succ_{i} y$ in $p$ to $x \sim_{i} y$ in $p^{\prime}$ (or the converse) can influence the global preference relation and cause a deterioration (or an improvement) of the global position of $x$, but not in all situations. That is why we speak of "limited influence."

Under Independence of Irrelevant Alternatives and Anonymity, Limited Influence of Indifference implies that, if $x \succsim^{n}(p) y$ and if we move parallel to the West border of the simplex, northward or southward, then we still have $x \succsim^{n}\left(p^{\prime}\right) y$ (see Fig.4). 

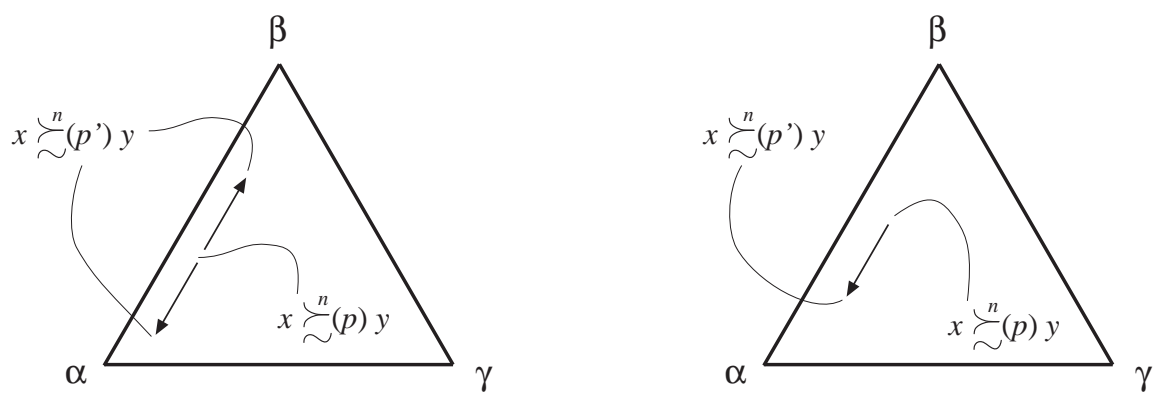

Figure 4: Geometrical representation of Limited Influence of Indifference (left) and Weak Non-Negative Responsiveness 2 (right).

Theorem 1 If a n-aggregation procedure satisfies Independence of Irrelevant Alternatives (A1), Anonymity (A2), Neutrality (A3), Weak Non-Negative Responsiveness 1 (A4), Unanimity (A5) and Limited Influence of Indifference (A6), then it is a generalized qualified weak majority.

Proof. By Independence of Irrelevant Alternatives and Anonymity, we know that the outcome between $x$ and $y$ depends only on $\alpha, \beta$ and $\gamma$. From here, we will abuse the notation and write, e.g. $x \succsim^{n}(\alpha, \beta, \gamma) y$ when we mean $x \succsim^{n}(p) y$ for all profiles $p$ such that $\alpha, \beta$ and $\gamma$ are the numbers of criteria such that, respectively, $x \succ_{i} y, x \sim_{i} y$ and $y \succ_{i} x$. By Unanimity, we know that there are points in the simplex such that $x \succsim^{n}(\alpha, \beta, \gamma) y$. Among these points, let $\alpha^{0}, \beta^{0}$ and $\gamma^{0}$ be one such that $\alpha+\beta$ is minimal. Then, using Weak Non-Negative Responsiveness 1 and Limited Influence of Indifference, we can move to any point $\left(\alpha^{\prime}, \beta^{\prime}, \gamma^{\prime}\right)$ in the simplex such that $\alpha^{\prime}+\beta^{\prime} \geq \alpha^{0}+\beta^{0}$ and we still have $x \succsim^{n}\left(\alpha^{\prime}, \beta^{\prime}, \gamma^{\prime}\right) y$. Hence, $x \succsim^{n}(\alpha, \beta, \gamma) y$ iff $\alpha+\beta \geq \alpha^{0}+\beta^{0}$. By Neutrality, we can permute $x$ and $y$ and we find that $y \succsim(\alpha, \beta, \gamma) x$ iff $\gamma+\beta \geq \alpha^{0}+\beta^{0}$. So, between $x$ and $y$, the $n$-procedure is a generalized qualified weak majority. Applying again Neutrality, we can generalize this conclusion to all pairs. The threshold $\delta_{n}$ is equal to $\alpha^{0}+\beta^{0}$.

If we want to avoid incomparability, we may use the following simple (but strong) condition.

A 7 Completeness. For all $x$ and $y$ in $X, x \succsim^{n}(p) y$ or $y \succsim^{n}(p) x$.

Theorem 2 If a n-aggregation procedure satisfies Independence of Irrelevant Alternatives (A1), Anonymity (A2), Neutrality (A3), Weak Non-Negative Responsiveness 1 (A4), Unanimity (A5), Limited Influence of Indifference (A6) and Completeness (A7), then it is a qualified weak majority.

Proof. By Theorem 1, the $n$-procedure is a generalized qualified weak majority. Then, by Completeness, for all $\alpha, \beta, \gamma$ such that $\alpha+\beta+\gamma=n$, we have $\alpha+\beta \geq \delta_{n}$ or $\beta+\gamma \geq \delta_{n}$. This is true, in particular, when $\beta=0$ and 
- $\alpha=\gamma=n / 2=\lceil n / 2\rceil(n$ even $)$

- $\alpha=(n+1) / 2=\lceil n / 2\rceil$ and $\gamma=(n-1) / 2(n$ odd $)$.

So, in both cases, $\delta_{n}$ can be at most $\lceil n / 2\rceil$.

A problem with the qualified weak majority is that many alternatives are globally indifferent, the $n$-procedure does not help to decide between them, because the indifference area is very large. So, we say that a $n$-procedure $\succsim^{n}$ is more decisive than another one $\succsim^{n \prime}$ if there are profiles such that $\succsim^{n}$ gives a strict preference and $\succsim^{n \prime}$ an indifference or an incomparability and if there are no profiles where $\succsim^{n \prime}$ yields a strict preference and $\succsim^{n}$ an indifference or an incomparability. So, we can easily characterize the weak majority in the following theorem (without proof).

Theorem 3 Among the n-aggregation procedures satisfying Independence of Irrelevant Alternatives (A1), Anonymity (A2), Neutrality (A3), Weak NonNegative Responsiveness 1 (A4), Unanimity (A5), Limited Influence of Indifference (A6) and Completeness (A7), the weak majority is the most decisive one.

Note that weak majority has been previously characterized by P. C. Fishburn [8]. The conditions he used are somehow similar to mine but they do not easily allow to present weak majority as a special case of generalized qualified weak majority.

Finally, in order to characterize ELECTRE I, I introduce

A 8 Restricted Positive Responsiveness. Suppose $p$ and $p^{\prime}$ are two identical profiles except that there is a criterion $i$ and two alternatives $x$ and $y$ such that $y \succ_{i} x$ and $x \succ_{i}^{\prime} y$. Suppose also that, for no criterion $i, x \sim_{i} y$. Then $x \sim(p) y$ implies $x \iota^{n}\left(p^{\prime}\right) y$.

Theorem 4 If a n-aggregation procedure satisfies Independence of Irrelevant Alternatives (A1), Anonymity (A2), Neutrality (A3), Weak Non-Negative Responsiveness 1 (A4), Unanimity (A5), Limited Influence of Indifference (A6) and Restricted Positive Responsiveness (A8), then it is an ELECTRE I method.

Note finally that, in most cases, ElECTRE I is used with a threshold larger than $n / 2$ and, so, it never happens that $x$ and $y$ are globally indifferent while there is no criterion where $x$ and $y$ are indifferent. Hence, Restricted Positive Responsiveness is trivially satisfied and does not really help us to understand how ELECTRE I works. It is therefore more interesting to characterise ELECTRE I with $\delta_{n}>n / 2$. That is why we introduce a new condition.

A 9 Minimal Incomparability. Suppose $n$ is even. There is at least one situation where $x$ and $y$ must be considered as incomparable: when the conflict is maximal, that is when $x$ is strictly better than $y$ for $n / 2$ criteria and $y$ is strictly better than $x$ for the other $n / 2$ criteria. But, because $n$ can be odd, we must 
adapt the condition for that case. The alternatives $x$ and $y$ must be incomparable if $x$ is strictly better than $y$ for $(n+1) / 2$ criteria and $y$ is strictly better than $x$ for the other $(n-1) / 2$ criteria, or the converse.

The meaning of this condition is clear. If we impose it, we adopt a prudent attitude. We avoid that the $n$-aggregation procedure always gives a clear-cut and easily interpretable result even when it (perhaps) should not. But the cost of imposing this condition is also clear. The global preference relation might be difficult to interpret or use. It is up to the decision-maker to choose between prudence and ease of interpretation.

Theorem 5 If a n-procedure satisfies Independence of Irrelevant Alternatives (A1), Anonymity (A2), Neutrality (A3), Weak Non-Negative Responsiveness 1 (A4), Unanimity (A5), Limited Influence of Indifference (A6) and Minimal Incomparability (A9), then it is an ELECTRE I method with $\delta_{n}>n / 2$.

\subsection{The a-qualified simple majority and some special cases}

Let us now present a condition that is satisfied by all $n$-procedures discussed in this paper and that will be useful in this section.

A 10 Weak Non-Negative Responsiveness 2. Suppose $p$ and $p^{\prime}$ are two identical profiles except that there is a criterion $i$ and two alternatives $x$ and $y$ such that $x \sim_{i} y$ and $x \succ_{i}^{\prime} y$. If $x \succsim^{n}(p) y$, then $x \succsim^{n}\left(p^{\prime}\right) y$.

This condition, like Weak Non-Negative Responsiveness 1 , is very compelling for it imposes that turning a single-criterion indifference $x \sim_{i} y$ into $x \succ_{i}^{\prime} y$ (thus improving the position of $x$ w.r.t. $y$ ) cannot lead to a deterioration of the position of $x$ w.r.t. $y$ in the global preference relation.

Under Independence of Irrelevant Alternatives and Anonymity, Weak NonNegative Responsiveness 2 implies that, if $x \succsim^{n}(p) y$ and if we move southward on a line parallel to the West border of the simplex, then we still have $x \succsim^{n}\left(p^{\prime}\right) y$ (see Fig.4). Note that Weak Non-Negative Responsiveness 2 is implied by Limited Influence of Indifference but the converse is not true.

The next condition is very specific of simple majority. It expresses the fact that two criteria (one for which $x \succ_{i} y$ and another one for which $y \succ_{j} x$ ) are equivalent to two criteria for which $x$ and $y$ are indifferent.

A 11 Pairwise Cancellation. Suppose $p$ and $p^{\prime}$ are two identical profiles except that there are two criteria $i, j$ and two alternatives $x$ and $y$ such that

- $x \succ_{i} y$ and $x \sim_{i}^{\prime} y$

- $y \succ_{j} x$ and $x \sim_{j}^{\prime} y$.

Then $x \succsim^{n}(p) y$ iff $x \succsim^{n}\left(p^{\prime}\right) y$. 

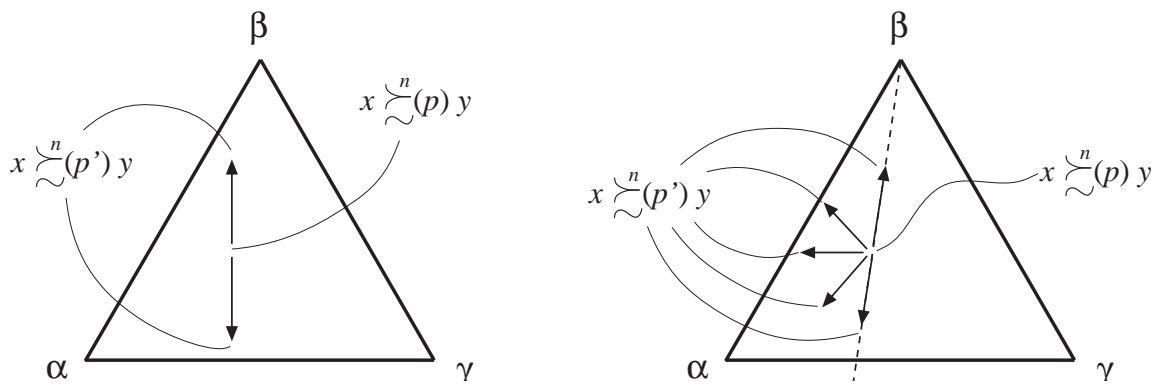

Figure 5: Geometrical representation of Pairwise Cancellation (left) and condition C (right).

Under Independence of Irrelevant Alternatives and Anonymity, Pairwise Cancellation implies that, if $x \succsim^{n}(p) y$ and if we move vertically in the simplex, northward or southward, then we still have $x \succsim^{n}\left(p^{\prime}\right) y$ (see Fig.5).

Theorem 6 If a n-aggregation procedure satisfies Independence of Irrelevant Alternatives (A1), Anonymity (A2), Neutrality (A3), Weak Non-Negative Responsiveness 2 (A10), Unanimity (A5) and Pairwise Cancellation (A11), then it is a a-qualified simple majority.

Proof. By Unanimity, we know that there are points in the simplex such that $x \succsim(\alpha, \beta, \gamma) y$ (same notation as in the proof of Theorem 1). Among these points, let $\alpha^{0}, \beta^{0}$ and $\gamma^{0}$ be one such that $\alpha-\gamma$ is minimal. Then, using Weak Non-Negative Responsiveness 2 and Pairwise Cancellation, we can move to any point in the simplex such that $\alpha^{\prime}-\gamma^{\prime} \geq \alpha^{0}-\gamma^{0}$ and we still have $x \succsim\left(\alpha^{\prime}, n-\alpha^{\prime}-\gamma^{\prime}, \gamma^{\prime}\right) y$. Hence,

$$
x \succsim(\alpha, \beta, \gamma) y \text { iff } \alpha-\gamma \geq \alpha^{0}-\gamma^{0} .
$$

If we let $\delta_{n}=\alpha^{0}-\gamma^{0}$, then we can rewrite the previous equivalence as

$$
\begin{array}{lll}
x \succsim(\alpha, \beta, \gamma) y & \text { iff } & \alpha \geq \gamma+\delta_{n} \\
& \text { iff } \quad \alpha+\beta \geq \gamma+\beta+\delta_{n}
\end{array}
$$

By Neutrality, we can permute $x$ and $y$ and we find that

$$
y \beth^{n}(\alpha, \beta, \gamma) x \text { iff } \gamma+\beta \geq \alpha+\beta+\delta_{n} .
$$

So, between $x$ and $y$, we have proved that the $n$-procedure is a qualified simple majority. Applying again Neutrality, we can generalize this conclusion to all pairs. By Unanimity and Neutrality, $-n<\delta_{n} \leq n$.

In the next theorem, we consider some special cases of a-qualified simple majority. 
Theorem 7 If a n-aggregation procedure satisfies Independence of Irrelevant Alternatives (A1), Anonymity (A2), Neutrality (A3), Weak Non-Negative Responsiveness 2 (A10), Unanimity (A5), Pairwise Cancellation (A11) and Completeness (A7), then it is a a-qualified simple majority with $\delta_{n} \leq 0$. Among these last n-procedures, simple majority is the most decisive one.

Completeness can be replaced by Reflexivity in Theorem 7 .

A 12 Reflexivity. The relation $\succsim^{n}(p)$ is reflexive for all $p$ in $\mathcal{C}^{n}$.

Geometrically, Reflexivity imposes that the top vertex of the simplex corresponds to an indifference.

It is worth recalling the first characterization of simple majority, by K. O. May. His result was relevant to the case of two alternatives. We present a straightforward generalization of his result for any finite number of alternatives. The only difference is the addition of Independence of Irrelevant Alternatives. We also make explicit some axioms that were part of his definition of a $n$ aggregation procedure.

A 13 Positive Responsiveness. Suppose $p$ and $p^{\prime}$ are two identical profiles except that there is a criterion $i$ and two alternatives $x$ and $y$ such that $\left[y \succ_{i} x\right.$ and $\left.x \succsim_{i}^{\prime} y\right]$ or $\left[y \sim_{i} x\right.$ and $\left.x \succ_{i}^{\prime} y\right]$. If $x \succsim^{n}(p) y$, then $x \succ^{n}\left(p^{\prime}\right) y$.

Theorem 8 ([12]) An aggregation procedure satisfies Independence of Irrelevant Alternatives (A1), Anonymity (A2), Neutrality (A3), Positive Responsiveness (A13) and Completeness (A7) iff it is simple majority.

We now turn to the case of a-qualified simple majority with $\delta \geq 0$.

Theorem 9 If a n-aggregation procedure satisfies Independence of Irrelevant Alternatives (A1), Anonymity (A2), Neutrality (A3), Weak Non-Negative Responsiveness 2 (A10), Unanimity (A5), Pairwise Cancellation (A11) and Restricted Positive Responsiveness (A8), then it is a a-qualified simple majority with $\delta_{n} \geq 0$.

Restricted Positive Responsiveness can of course be replaced by Minimal Incomparability in this theorem, but, then, $\delta_{n}>0$.

\subsection{Tactic majority, Generalized r-Tactic majority and m- qualified simple majority}

Up to now, we characterized various $n$-aggregation procedures by adding one or two conditions to Independence of Irrelevant Alternatives, Anonymity, Neutrality, Unanimity, Weak Non-Negative Responsiveness 1 and 2. We can do the same for TACTIC majority, Generalized r-TACTIC majority and m-qualified simple majority. But, unfortunately, the conditions that we must use to characterize these $n$-procedures are then very strong and not easily interpretable. Below, we give an example of such a characterization. 
A 14 Condition C. Suppose $p$ and $p^{\prime}$ are two identical profiles of size $n$ except that there are two sets of criteria $I=\left\{i_{1}, \ldots, i_{\mu}\right\}$ and $J=\left\{j_{1}, \ldots, j_{\nu}\right\}$ and two alternatives $x$ and $y$ such that

- $x \succ_{i} y$ and $x \sim_{i}^{\prime} y$ for all $i$ in $I$

- $y \succ_{j} x$ and $x \sim_{j}^{\prime} y$ for all $j$ in $J$.

Then

$$
\frac{\mu}{\nu} \leq \frac{\#\left\{i \in N: x \succ_{i} y\right\}}{\#\left\{j \in N: y \succ_{j} x\right\}} \quad \text { implies } \quad\left[x \succsim^{n}(p) y \Rightarrow x \succsim^{n}\left(p^{\prime}\right) y\right]
$$

and

$$
\frac{\mu}{\nu} \geq \frac{\#\left\{i \in N: x \succ_{i} y\right\}}{\#\left\{j \in N: y \succ_{j} x\right\}} \quad \text { implies } \quad\left[x \succsim^{n}\left(p^{\prime}\right) y \Rightarrow x \succsim^{n}(p) y\right] .
$$

Under Independence of Irrelevant Alternatives and Anonymity, Relative Pairwise Cancellation implies that, if $x \succsim^{n}(p) y$ and if we move to any point West of the straight line passing through the top vertex of the simplex and $p$, then we still have $x \succsim^{n}\left(p^{\prime}\right) y$ (see Fig.5). Remark that condition C implies Weak NonNegative Responsiveness 1 and 2.

Theorem 10 If a n-aggregation procedure satisfies Independence of Irrelevant Alternatives (A1), Anonymity (A2), Neutrality (A3), Unanimity (A5) and Condition $C$ (A14), then it is a generalized $r$-TACTIC majority.

Proof. By Unanimity, we know that there are points in the simplex such that $x \succsim^{n}(\alpha, \beta, \gamma) y$ (same notation as in the proof of Theorem 1). Among these points, let $\alpha^{0}, \beta^{0}$ and $\gamma^{0}$ be one such that $\alpha / \gamma$ is minimal. Then, using Condition C, we can move to any point in the simplex such that $\alpha^{\prime} / \gamma^{\prime} \geq \alpha^{0} / \gamma^{0}$ and we still have $x \succsim\left(\alpha^{\prime}, n-\alpha^{\prime}-\gamma^{\prime}, \gamma^{\prime}\right) y$. Hence,

$$
x \succsim^{n}(\alpha, \beta, \gamma) y \text { iff } \alpha / \gamma \geq \alpha^{0} / \gamma^{0} .
$$

If we let $\delta=\alpha^{0} / \gamma^{0}$, then we can rewrite the previous equivalence as

$$
x \succsim(\alpha, \beta, \gamma) y \text { iff } \alpha \geq \gamma \delta .
$$

By Neutrality, we can permute $x$ and $y$ and we find that

$$
y \succsim(\alpha, \beta, \gamma) x \text { iff } \gamma \geq \alpha \delta
$$

So, between $x$ and $y$, we have proved that the $n$-procedure is a generalized $\mathrm{r}$ TACTIC majority. Applying again Neutrality, we can generalize this conclusion to all pairs. By Unanimity, $0<\delta \leq n$.

A very simple result follows (without proof).

Theorem 11 If a n-aggregation procedure satisfies Independence of Irrelevant Alternatives (A1), Anonymity (A2), Neutrality (A3), Unanimity (A5), Condition C (A14) and Minimal Incomparability (A9), then it is a r-TACTIC majority. 
Because the conditions we must use for characterizing TACTIC majority, Generalized r-TACTIC majority and m-qualified simple majority are very strong, we do not devote much place to them. We will see more interesting results about these majorities in the next section when we consider aggregation procedures and no more $n$-aggregation procedures.

\section{Characterization of some aggregation proce- dures}

In this section, we will consider that $n$, the number of criteria, can vary. There is a priori no link between the different $n$-procedures in an aggregation procedure.

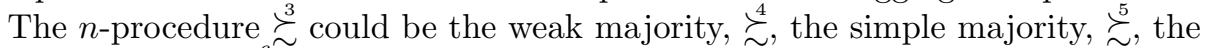
Borda rule and $\succsim$, still another $n$-procedure.

A key property in the analysis of $n$-aggregation procedures is Homogeneity. It will impose that an aggregation procedure be the same for different $n$ in the following sense.

A 15 Homogeneity. Suppose that $p=\left(\succsim_{1}, \succsim_{2}, \ldots, \succsim_{n}\right)$ and

$$
p^{\prime}=(\underbrace{\succsim_{1}, \ldots, \succsim_{1}}_{m}, \ldots, \underbrace{\succsim_{n}, \ldots, \succsim_{n}}_{m}) .
$$

Then $x \succsim^{n}(p) y$ iff $x \searrow^{r}\left(p^{\prime}\right) y$, with $r=m n$.

\subsection{Some trivial results}

All theorems of Section 3 can be trivially extended to aggregation procedures. We just need to impose the axioms for all $n$ in $\mathbb{N}$. And if we want that the threshold (in terms of proportions) be (almost) identical for all $n$, then we just need to impose Homogeneity. We now present the extension of theorem 1 as an example. The extension of the other theorems of Section 3 is left to the reader.

Theorem 12 If an aggregation procedure $\succsim=\left\{\succsim^{1}, \ldots, \succsim^{n}, \ldots\right\}$ satisfies Homogeneity (A15) and, for all $n$ in $\mathbb{N}$, Independence of Irrelevant Alternatives (A1), Anonymity (A2), Neutrality (A3), Weak Non-Negative Responsiveness 1 (A4), Unanimity (A5) and Limited Influence of Indifference (A6), then $\succsim^{n}$ is a generalized qualified weak majority for all $n$ in $\mathbb{N}$. Furthermore, there exists $\dot{\delta}$ in $\mathbb{Q}$ such that $\delta_{n}=\lceil n \dot{\delta}\rceil$, for all $n$ in $\mathbb{N}$.

Proof. By Theorem 1, each $n$-aggregation procedure is a generalized qualified weak majority, with $\delta_{n}$. So we just need to prove that there exists $\dot{\delta}$ in $\mathbb{Q}$ such that $\delta_{n}=\lceil n \dot{\delta}\rceil$, for all $n$ in $\mathbb{N}$. Suppose it is not the case; there are then $n$ and $n^{\prime}$ such that $\delta_{n} / n \leq\left(\delta_{n^{\prime}}-1\right) / n^{\prime}$ or $\delta_{n^{\prime}} / n^{\prime} \leq\left(\delta_{n}-1\right) / n$. Without loss of generality, assume $\delta_{n} / n \leq\left(\delta_{n^{\prime}}-1\right) / n^{\prime}$. By Homogeneity, $n\left(\delta_{n^{\prime}}-1\right)<\delta_{n n^{\prime}} \leq n \delta_{n^{\prime}}$. So,

$$
\frac{\delta_{n^{\prime}}-1}{n^{\prime}}<\frac{\delta_{n n^{\prime}}}{n n^{\prime}} \leq \frac{\delta_{n^{\prime}}}{n^{\prime}} .
$$


Similarly,

$$
\frac{\delta_{n}-1}{n}<\frac{\delta_{n n^{\prime}}}{n n^{\prime}} \leq \frac{\delta_{n}}{n}
$$

Hence,

$$
\frac{\delta_{n^{\prime}}-1}{n^{\prime}}<\frac{\delta_{n}}{n}
$$

This contradicts the inequality we found above and the proof is complete.

In the next subsections, we present some results in which Homogeneity plays a central role, allowing us to characterize TACTIC majority, Generalized rTACTIC majority and m-qualified simple majority using much weaker conditions than in section 3.3 .

\subsection{Tactic and Generalized $r$-Tactic majority}

A necessary condition for TACTIC and Generalized $r$-TACTIC majority is

A $16 \succ$-Invariance. Let $p$ and $p^{\prime}$ be two profiles such that

$$
\frac{\#\left\{i \in N: x \succ_{i} y\right\}}{\#\left\{i \in N: y \succ_{i} x\right\}}=\frac{\#\left\{i \in N: x \succ_{i}^{\prime} y\right\}}{\#\left\{i \in N: y \succ_{i}^{\prime} x\right\}} .
$$

Then $x \succsim^{n}(p) y$ iff $x \succsim^{n}\left(p^{\prime}\right) y$.

This condition states that what matters for deciding whether $x \succsim_{\sim}^{n}(p) y$ is the ratio of the number of criteria strictly supporting $x$ against $y$ (i.e. $x \succ_{i} y$ ) and of those strictly supporting $y$ against $x$.

Under Independence of Irrelevant Alternatives and Anonymity, $\succ$-Invariance implies that, if $x \succsim^{n}(p) y$ and if we move in the simplex along a line passing through the North vertex, northward or southward, then we still have $x_{\beth^{n}}^{n}\left(p^{\prime}\right) y$ (see Fig.6).

Another necessary condition for TACTIC is

A 17 Asymmetry. The relation $\succsim^{n}(p)$ is asymmetric.

Asymmetry and $\succ$-Invariance are obviously necessary for TACTIC majority. But they are not sufficient (with Independance of Irrelevant Alternatives, Anonymity, Neutrality, Weak Non-Negative Responsiveness 1 or 2 and Unanimity) to derive the Generalized $r$-TACTIC majority, as shown in Fig.6. The 5-procedure represented in this figure has all the properties listed before but is not a TACTIC majority. Nevertheless, if we add Homogeneity to the previous conditions, then we obtain a TACTIC majority.

Theorem 13 If an aggregation procedure $\succsim=\left\{\succsim^{1}, \ldots, \succsim^{n}, \ldots\right\}$ satisfies Homogeneity (A15) and, for all $n$ in $\mathbb{N}$, Independence of Irrelevant Alternatives (A1), Anonymity (A2), Neutrality (A3), Weak Non-Negative Responsiveness 2 (A10), Unanimity (A5), Asymmetry (A17) and $\succ$-Invariance (A16), then $\succsim^{n}$ is TACTIC majority for all $n$ in $\mathbb{N}$. Furthermore, there exists $\dot{\delta}$ in $\mathbb{Q}$ such that, for all $n$ in $\mathbb{N}, k \delta_{n}=\lceil k \dot{\delta}\rceil$, for some $k$ in $N$. 

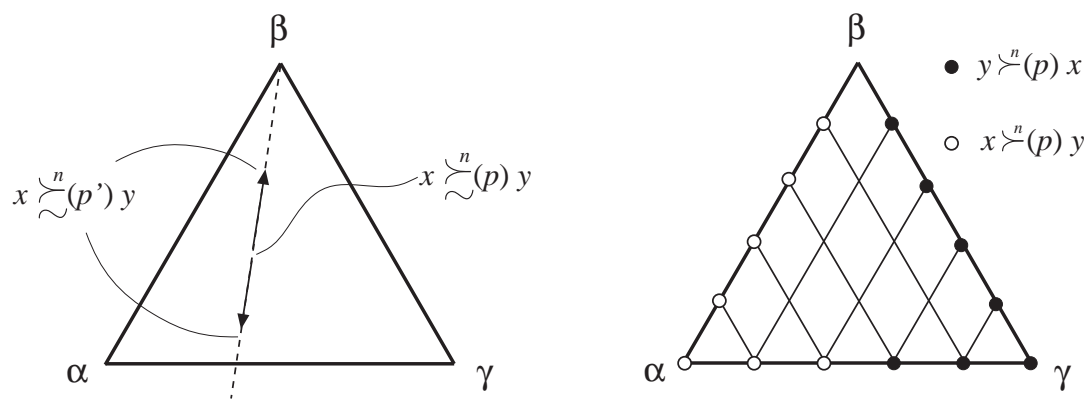

Figure 6: Left: geometrical representation of $\succ$-Invariance. Right: a 5procedure satisfying Independance of Irrelevant Alternatives, Anonymity, Neutrality, Weak Non-Negative Responsiveness 1 and 2, Unanimity and $\succ$ Invariance but which is not a TACTIC majority. The points in the simplex without any dot (empty or filled) correspond to profiles such that $x$ and $y$ are incomparable.

Proof. Let us first fix $n$. By Independence of Irrelevant Alternatives and Anonymity, we know that the outcome between $x$ and $y$ depends only on $\alpha_{n}, \beta_{n}$ and $\gamma_{n}$ (we now use a subscript $n$ because we will use, further in this proof, a profile of another size). From here, we will abuse the notation and write, e.g. $x \succsim^{n}\left(\alpha_{n}, \beta_{n}, \gamma_{n}\right) y$ when we mean $x \succsim^{n}(p) y$ for all profiles $p$ of size $n$ such that $\alpha_{n}, \beta_{n}$ and $\gamma_{n}$ are the numbers of criteria such that, respectively, $x \succ_{i} y, x \sim_{i} y$ and $y \succ_{i} x$. By Unanimity, we know that there are points in the simplex such that $x \succsim\left(\alpha_{n}, \beta_{n}, \gamma_{n}\right) y$. Among these points, let $\alpha_{n}^{0}, \beta_{n}^{0}$ and $\gamma_{n}^{0}$ be one such that $\alpha_{n} / \gamma_{n}$ is minimal. Consider any point in the simplex such that $\alpha_{n}^{\prime} / \gamma_{n}^{\prime} \geq \alpha_{n}^{0} / \gamma_{n}^{0}$. If $\alpha_{n}^{\prime} / \gamma_{n}^{\prime}=\alpha_{n}^{0} / \gamma_{n}^{0}$, then we can reach it making one of the moves described in $\succ$-Invariance. If $\alpha_{n}^{\prime} / \gamma_{n}^{\prime}>\alpha_{n}^{0} / \gamma_{n}^{0}$, then, in some cases, it is possible to reach it by combining some of the moves described in $\succ$-Invariance and Weak NonNegative Responsiveness 2. But, sometimes, it is not possible. For an example, look at Fig.6. In this case, $\alpha_{5}^{0}=3$ and $\gamma_{5}^{0}=2$. From this point, it is not possible to reach $\left(\alpha_{5}^{\prime}=3, \gamma_{5}^{\prime}=1\right)$ using $\succ$-Invariance and Weak Non-Negative Responsiveness 2 although $\alpha_{5}^{\prime} / \gamma_{5}^{\prime}>\alpha_{5}^{0} / \gamma_{5}^{0}$. Even if we would use Weak NonNegative Responsiveness 1, it would not be possible.

Consider now the ratio $\gamma_{n}^{\prime} \alpha_{n}^{0} / \gamma_{n}^{0}$. It is a rational number. Let us call $m$ the smallest integer such that $m \gamma_{n}^{\prime} \alpha_{n}^{0} / \gamma_{n}^{0}$ is integer. Set $\alpha_{m n}^{*}=m \gamma_{n}^{\prime} \alpha_{n}^{0} / \gamma_{n}^{0}, \gamma_{m n}^{*}=$ $m \gamma_{n}^{\prime}$ and $\beta_{m n}^{*}=m n-\gamma_{m n}^{*}-\alpha_{m n}^{*}$. By Homogeneity, $x \succsim_{\sim}^{m n}\left(m \alpha_{n}^{0}, m \beta_{n}^{0}, m \gamma_{n}^{0}\right) y$. By $\succ$-Invariance, $x \succsim^{m n}\left(\alpha_{m n}^{*}, \beta_{m n}^{*}, \gamma_{m n}^{*}\right) y$ since $\alpha_{m n}^{*} / \gamma_{m n}^{*}=\alpha_{n}^{0} / \gamma_{n}^{0}$. Then, by Weak Non-Negative Responsiveness 2, $x \succsim^{m n}\left(m \alpha_{n}^{\prime}, m \beta_{n}^{\prime}, m \gamma_{n}^{\prime}\right) y$ since $m \gamma_{n}^{\prime}=\gamma_{m n}^{*}$ and $m \alpha_{n}^{\prime}>\alpha_{m n}^{*}$. Finally, by Homogeneity, $x \succsim_{\sim}^{n}\left(\alpha_{n}^{\prime}, \beta_{n}^{\prime}, \gamma_{n}^{\prime}\right) y$.

So, using $\succ$-Invariance and Weak Non-Negative responsiveness 2 , we can move to any point in the simplex such that $\alpha_{n}^{\prime} / \gamma_{n}^{\prime} \geq \alpha_{n}^{0} / \gamma_{n}^{0}$ and we still have $x \succsim\left(\alpha_{n}^{\prime}, n-\alpha_{n}^{\prime}-\gamma_{n}^{\prime}, \gamma_{n}^{\prime}\right) y$. Hence,

$$
x \succsim^{n}\left(\alpha_{n}, \beta_{n}, \gamma_{n}\right) y \text { iff } \alpha_{n} / \gamma_{n} \geq \alpha_{n}^{0} / \gamma_{n}^{0} .
$$


If we let $\delta_{n}^{\prime}=\alpha_{n}^{0} / \gamma_{n}^{0}$, then we can rewrite the previous equivalence as

$$
x \succsim^{n}\left(\alpha_{n}, \beta_{n}, \gamma_{n}\right) y \text { iff } \alpha_{n} \geq \gamma_{n} \delta_{n}^{\prime} .
$$

By Neutrality, we can permute $x$ and $y$ and we find that

$$
y \succsim\left(\alpha_{n}, \beta_{n}, \gamma_{n}\right) x \text { iff } \gamma_{n} \geq \alpha_{n} \delta_{n}^{\prime} .
$$

By Asymmetry, we know that $x$ and $y$ are incomparable in $\succsim^{n}(p)$ when $\beta=n$. Using again Asymmetry, we find that $\delta_{n}^{\prime}>1$ and, hence, we can rewrite (1) as

$$
x \overbrace{}^{n}\left(\alpha_{n}, \beta_{n}, \gamma_{n}\right) y \text { iff } \alpha_{n}>\gamma_{n} \delta_{n},
$$

where $\delta_{n}$ is the largest number such that $\delta_{n}<\delta_{n}^{\prime}$ and $i \delta_{n} \in N$, for some $i \in N$. So, between $x$ and $y$, we have proved that the $n$-procedure is TACTIC majority. Applying again Neutrality, we can generalize this conclusion to all pairs. By Unanimity, $1 \leq \delta_{n}<n$.

Following the same reasoning as in Theorem 12, we find that there exists $\dot{\delta}$ in $\mathbb{Q}$ such that, for all $n$ in $\mathbb{N}, k \delta_{n}=\lceil k \dot{\delta}\rceil$, for some $k$ in $N$.

It is clear that we can prove a similar theorem replacing Weak Non-Negative responsiveness 2 by Weak Non-Negative responsiveness 1 .

Let us now consider the Generalized $r$-TACTIC majority.

Theorem 14 If an aggregation procedure $\succsim=\left\{\succsim^{1}, \ldots, \succsim^{n}, \ldots\right\}$ satisfies Homogeneity (A15) and, for all $n$ in $\mathbb{N}$, Independence of Irrelevant Alternatives (A1), Anonymity (A2), Neutrality (A3), Weak Non-Negative Responsiveness 2 (A10), Unanimity (A5), Reflexivity (A12) and $\succ$-Invariance (A16), then $\succsim^{n}$ is a Generalized $r$-TACTIC majority for all $n$ in $\mathbb{N}$. Furthermore, there exists $\dot{\delta}$ in $\mathbb{Q}$ such that, for all $n$ in $\mathbb{N}, k \delta_{n}=\lceil k \dot{\delta}\rceil$, for some $k$ in $N$.

The proof is almost the same as that of Theorem 13 except that we use Reflexivity instead of Asymmetry at the end of the proof.

We now state a theorem characterizing different particular cases of Generalized $r$-TACTIC majority.

Theorem 15 An aggregation procedure $\succsim=\left\{\succsim^{1}, \ldots, \succsim^{n}, \ldots\right\}$ satisfies Homogeneity (A15) and, for all $n$ in $\mathbb{N}$, Independence of Irrelevant Alternatives (A1), Anonymity (A2), Neutrality (A3), Weak Non-Negative Responsiveness 2 (A10), Unanimity (A5), $\succ$-Invariance (A16) and Completeness (A7) if and only if $\succsim^{n}$ is a Generalized $r$-TACTIC majority with $\dot{\delta} \leq 1$. If we replace Completeness by Restricted Positive responsiveness and Reflexivity, then $\dot{\delta} \geq 1$ and if we replace it by Minimal Incomparability and Reflexivity, then $\dot{\delta}>1$.

\subsection{The m-qualified simple majority}

A necessary condition for m-qualified simple majority is 
A $18 \succsim$-Invariance. Let $p$ and $p^{\prime}$ be two profiles such that

$$
\frac{\#\left\{i \in N: x \succsim_{i} y\right\}}{\#\left\{i \in N: y \succsim_{i} x\right\}}=\frac{\#\left\{i \in N: x \succsim_{i}^{\prime} y\right\}}{\#\left\{i \in N: y \succsim_{i}^{\prime} x\right\}} .
$$

Then $x \succsim^{n}(p) y$ iff $x \succsim^{n}\left(p^{\prime}\right) y$.

This condition states that what matters for deciding whether $x \succsim^{n}(p) y$ is the ratio of the number of criteria weakly supporting $x$ against $y$ (i.e. $x \succsim_{i} y$ ) and of those weakly supporting $y$ against $x$.

Under Independence of Irrelevant Alternatives and Anonymity, $\succsim$-Invariance implies that, if $x \succsim^{n}(p) y$ and if we move in the simplex along a line passing by a point symmetrical to the North vertex, northward or southward, then we still have $x \succsim^{n}\left(p^{\prime}\right) y$ (see Fig.7).

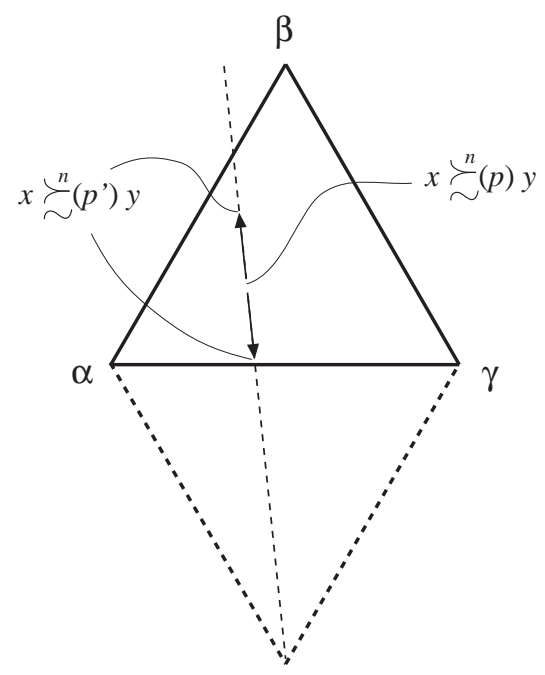

Figure 7: Geometrical representation of $\succsim$-Invariance.

This condition is obviously necessary for a m-qualified simple majority. As for m-qualified simple majority, $\succsim$-Invariance (combined with Independance of Irrelevant Alternatives, Anonymity, Neutrality, Unanimity and Weak NonNegative Responsiveness 1 or 2) is not enough to derive the m-qualified simple majority. Nevertheless, if we add Homogeneity to the previous conditions, the we obtain a m-qualified simple majority.

Theorem 16 If an aggregation procedure $\succsim=\left\{\succsim^{1}, \ldots, \succsim^{n}, \ldots\right\}$ satisfies Homogeneity (A15) and, for all $n$ in $\mathbb{N}$, Independence of Irrelevant Alternatives (A1), Anonymity (A2), Neutrality (A3), Weak Non-Negative Responsiveness 2 (A10), Unanimity (A5) and $\succsim$-Invariance. (A18), then $\succsim^{n}$ is a m-qualified simple majority for all $n$ in $\mathbb{N}$. Furthermore, there exists $\dot{\delta}$ in $\mathbb{Q}$ such that, for all $n$ in $\mathbb{N}$, $k \delta_{n}=\lceil k \dot{\delta}\rceil$, for some $k$ in $N$. 
Proof. By Unanimity, we know that there are points in the simplex such that $x \succsim\left(\alpha_{n}, \beta_{n}, \gamma_{n}\right) y$ (same notation as in the proof of Theorem 13). Among these points, let $\alpha_{n}^{0}, \beta_{n}^{0}$ and $\gamma_{n}^{0}$ be one such that

$$
\frac{\alpha_{n}+\beta_{n}}{\gamma_{n}+\beta_{n}}
$$

is minimal. Consider any point in the simplex such that

$$
\frac{\alpha_{n}^{\prime}+\beta_{n}^{\prime}}{\gamma_{n}^{\prime}+\beta_{n}^{\prime}} \geq \frac{\alpha_{n}^{0}+\beta_{n}^{0}}{\gamma_{n}^{0}+\beta_{n}^{0}}
$$

If

$$
\frac{\alpha_{n}^{\prime}+\beta_{n}^{\prime}}{\gamma_{n}^{\prime}+\beta_{n}^{\prime}}=\frac{\alpha_{n}^{0}+\beta_{n}^{0}}{\gamma_{n}^{0}+\beta_{n}^{0}}
$$

then we can reach it making one of the moves described in $\succsim$-Invariance. If

$$
\frac{\alpha_{n}^{\prime}+\beta_{n}^{\prime}}{\gamma_{n}^{\prime}+\beta_{n}^{\prime}}>\frac{\alpha_{n}^{0}+\beta_{n}^{0}}{\gamma_{n}^{0}+\beta_{n}^{0}}
$$

then, in some cases, it is possible to reach it by combining some of the moves described in $\succsim$-Invariance and Weak Non-Negative Responsiveness 2. But, sometimes, it is not possible. For an example, look at Fig.6. In this case, $\alpha_{5}^{0}=3$ and $\gamma_{5}^{0}=2$. From this point, it is not possible to reach $\left(\alpha_{5}^{\prime}=3, \gamma_{5}^{\prime}=1\right)$ using $\succsim$-Invariance and Weak Non-Negative Responsiveness 2 although

$$
\frac{\alpha_{5}^{\prime}+\beta_{5}^{\prime}}{\gamma_{5}^{\prime}+\beta_{5}^{\prime}}>\frac{\alpha_{5}^{0}+\beta_{5}^{0}}{\gamma_{5}^{0}+\beta_{5}^{0}}
$$

Even if we would use Weak Non-Negative Responsiveness 1, it would not be possible.

Let $\delta_{n}$ denote

$$
\frac{\left(\alpha_{n}^{0}+\beta_{n}^{0}\right)}{\left(\gamma_{n}^{0}+\beta_{n}^{0}\right)}
$$

and let us call $m$ the smallest integer such that

$$
m\left(\frac{\gamma_{n}^{\prime}}{\delta_{n}}-\frac{n}{\delta_{n}}+n\right)
$$

is integer. Set

$$
\alpha_{m n}^{*}=m\left(\frac{\gamma_{n}^{\prime}}{\delta_{n}}-\frac{n}{\delta_{n}}+n\right)
$$

$\gamma_{m n}^{*}=m \gamma_{n}^{\prime}$ and $\beta_{m n}^{*}=m n-\gamma_{m n}^{*}-\alpha_{m n}^{*}$. By Homogeneity, $x \succsim^{m n}\left(m \alpha_{n}^{0}, m \beta_{n}^{0}, m \gamma_{n}^{0}\right) y$.

By $\succsim$-Invariance, $x \succsim^{m n}\left(\alpha_{m n}^{*}, \beta_{m n}^{*}, \gamma_{m n}^{*}\right) y$ since

$$
\frac{\alpha_{m n}^{*}+\beta_{m n}^{*}}{\gamma_{m n}^{*}+\beta_{m n}^{*}}=\frac{\alpha_{n}^{0}+\beta_{n}^{0}}{\gamma_{n}^{0}+\beta_{n}^{0}}=\delta_{n}
$$


Then, by Weak Non-Negative Responsiveness $2, x \succsim^{m n}\left(m \alpha_{n}^{\prime}, m \beta_{n}^{\prime}, m \gamma_{n}^{\prime}\right) y$ since $m \gamma_{n}^{\prime}=\gamma_{m n}^{*}$ and $m \alpha_{n}^{\prime}>\alpha_{m n}^{*}$. Finally, by Homogeneity, $x \succsim\left(\alpha_{n}^{\prime}, \beta_{n}^{\prime}, \gamma_{n}^{\prime}\right) y$.

So, using $\succsim$-Invariance and Weak Non-Negative responsiveness 2 , we can move to any point in the simplex such that

$$
\frac{\alpha_{n}^{\prime}+\beta_{n}^{\prime}}{\gamma_{n}^{\prime}+\beta_{n}^{\prime}} \geq \delta_{n}
$$

and we still have $x \succsim^{n}\left(\alpha_{n}^{\prime}, \beta_{n}^{\prime}, \gamma_{n}^{\prime}\right) y$. Hence,

$$
x \succsim\left(\alpha_{n}, \beta_{n}, \gamma_{n}\right) y \text { iff } \alpha_{n}+\beta_{n} \geq\left(\gamma_{n}+\beta_{n}\right) \delta_{n} .
$$

By Neutrality, we can permute $x$ and $y$ and we find that

$$
y \succsim^{n}\left(\alpha_{n}, \beta_{n}, \gamma_{n}\right) x \text { iff } \gamma_{n}+\beta_{n} \geq\left(\alpha_{n}+\beta_{n}\right) \delta_{n} .
$$

So, between $x$ and $y$, we have proved that the $n$-procedure is a m-qualified simple majority. Applying again Neutrality, we can generalize this conclusion to all pairs. By Unanimity, $0<\delta_{n} \leq n$.

Following the same reasoning as in Theorem 12, we find that there exists $\dot{\delta}$ in $\mathbb{Q}$ such that, for all $n$ in $\mathbb{N}, k \delta_{n}=\lceil k \dot{\delta}\rceil$, for some $k$ in $N$.

It is clear that we can prove a similar theorem replacing Weak Non-Negative responsiveness 2 by Weak Non-Negative responsiveness 1 .

\section{Conclusion}

All $n$-aggregation procedures we have discussed in sections 2 and 3 share the following properties: Independence of Irrelevant Alternatives, Anonymity, Neutrality, Unanimity, Weak Non-Negative Responsiveness 1 and 2. The corresponding aggregation procedures satisfy the same properties plus Homogeneity. What makes them different? We summarize below the conditions that allow us to characterize them.

- Generalized Qualified Weak Majority: Limited Influence of Indifference.

- a-Qualified Simple Majority: Pairwise cancellation.

- TACTIC Majority: $\succ$-Invariance and Asymmetry.

- Generalized r-TACTIC Majority: $\succ$-Invariance and Reflexivity.

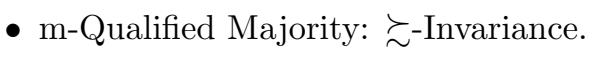

Within each of these families of procedures, it is possible to characterize some special cases, using Completeness, Reflexivity, Restricted Positive Responsiveness or Minimal Incomparability. 
Some characterizations of majority procedures existed in the literature but were not always easy to compare. In this paper, the main variants of anonymous and neutral majority have been characterized in a uniform way.

Of course, not all variants of majority have been discussed. For instance, nothing was said about non-neutral majorities (not so important in multicriteria decision aiding) and non-anonymous majorities (e.g. weighted majority). A uniform analysis of non-anonymous majorities would be more relevant to multicriteria decision aiding than this paper but I hope this paper can be seen as a first step in that direction.

Another limitation of this paper is that we supposed that the preference relation along each criterion is complete. This is of course not always the case. A generalization encompassing incompleteness is thus needed.

\section{References}

[1] G. Aşan and M. R. Sanver. Another characterization of the majority rule. Economics Letters, 75:409-413, 2002.

[2] N. Belacel and M. Boulassel. Multicriteria fuzzy classification procedure PROCFTN: Methodology and medical application. Fuzzy Sets and Systems, 141:203-217, 2004.

[3] D. E. Campbell and J. S. Kelly. A simple characterization of majority rule. Economic Theory, 15:689-700, 2000.

[4] F. Chiclana, F. Herrera, and E. Herrera-Viedma. Integrating multiplicative preference relations in a multipurpose decision-making model based on fuzzy preference relations. Fuzzy Sets and Systems, 122(2):277-291, 2001.

[5] L. C. Dias and J. N. Clímaco. Dealing with imprecise information in group multicriteria decisions: a methodology and a gdss architecture. European Journal of Operational Research, 160(2):291-307, 2005.

[6] D. Felsenthal and M. Machover. Ternary voting games. International Journal of Game Theory, 26:335-351, 1997.

[7] E. Fernandez and R. Olmedo. An agent model based on ideas of concordance and discordance for group ranking problems. Decision Support Systems, 39(3):429-443, May 2005.

[8] P. C. Fishburn. The theory of social choice. Princeton University Press, 1973.

[9] W. J. Hurley. Effects of multiple arbitrators on final-offer arbitration settlements. European Journal of Operational Research, 145:660-664, 2003.

[10] J. P. Leclercq. Propositions d'extension de la notion de dominance en présence de relations d'ordre sur les pseudo-critères : Melchior. Revue Belge 
de Recherche Opérationnelle, de Statistique et d'Informatique, 24(1):32-46, 1984.

[11] J. R. Marsden and S. Mathiyalakan. An investigation of changes in attitude over time of gdss groups under unanimity and majority decision rules. European Journal of Operational Research, 145(3):693-712, 2003.

[12] K. O. May. A set of independent necessary and sufficient conditions for simple majority decisions. Econometrica, 20:680-684, 1952.

[13] S. Oliveira and P. Ferreira. Bi-objective optimisation with multiple decision-makers: a convex approach to attain majority solutions. Journal of the Operational Research Society, 51(3):330-340, 2000.

[14] S. Opricovic and G.-H. Tzeng. Compromise solution by MCDM methods: A comparative analysis of VIKOR and TOPSIS. European Journal of Operational Research, 156(2):445-455, 2004.

[15] B. Roy. Classement et choix en présence de points de vue multiples (la méthode ELECTRE). Revue Française d'informatique et de recherche opérationnelle, 8:57-75, 1968.

[16] B. Roy and D. Bouyssou. Aide Multicritère à la Décision : Méthodes et Cas. Economica, Paris, 1993.

[17] D. G. Saari. Geometry of voting. Springer-Verlag, 1994.

[18] A. Schärlig. Décider sur plusieurs critères : panorama de l aide à la décision multicritère. Presses polytechniques et universitaires romandes, Lausanne, 1990.

[19] J. C. Vansnick. On the problem of weights in multiple criteria decision making (the non-compensatory approach). European Journal of Operational Research, 24:288-294, 1986.

[20] J. C. Vansnick. Principes et applications des méthodes multicritères. Technical report, Universit de Mons-Hainaut, 1988. 